Correspondence

Abdul N. Hamood

abdul.hamood@ttuhsc.edu

Received 27 October 2006

Accepted 5 March 2007

\section{Diversity of biofilms produced by quorum-sensing- deficient clinical isolates of Pseudomonas aeruginosa}

\author{
J. Andy Schaber, ${ }^{1,2} \dagger$ Adrienne Hammond, ${ }^{1,2}$ Nancy L. Carty, ${ }^{1}$ \\ Simon C. Williams, ${ }^{3}$ Jane A. Colmer-Hamood, ${ }^{1}$ Ben H. Burrowes, ${ }^{1}$ \\ Vijian Dhevan, ${ }^{4}$ John A. Griswold ${ }^{1,2}$ and Abdul N. Hamood ${ }^{1,2}$ \\ ${ }^{1}$ Department of Microbiology and Immunology, Texas Tech University Health Sciences Center, \\ Lubbock, TX 79430, USA \\ ${ }^{2}$ Department of Surgery, Texas Tech University Health Sciences Center, Lubbock, TX 79430, USA \\ ${ }^{3}$ Department of Cell Biology and Biochemistry, Texas Tech University Health Sciences Center, \\ Lubbock, TX 79430, USA \\ ${ }^{4}$ School of Medicine, Texas Tech University Health Sciences Center, Lubbock, TX 79430, USA
}

The quorum-sensing (OS) systems control several virulence attributes of Pseudomonas aeruginosa. Five QS-deficient $P$. aeruginosa clinical isolates $(\mathrm{Cl})$ that were obtained from wound $(\mathrm{Cl}-1)$, tracheal $(\mathrm{Cl}-2, \mathrm{Cl}-3, \mathrm{Cl}-4)$ and urinary tract $(\mathrm{Cl}-5)$ infections had previously been characterized. In this study, a flow-through continuous-culture system was utilized to examine in detail the biofilms formed by these isolates in comparison with the $P$. aeruginosa prototrophic strain PAO1. Analysis of the biofilms by confocal laser scanning microscopy and COMSTAT image analysis at 1 and 7 days post-inoculation showed that the isolates produced diverse biofilms. In comparison with PAO1, the $\mathrm{Cl}$ produced biofilms that scarcely or partially covered the surface at day 1 , although $\mathrm{Cl}-1$ produced larger microcolonies. At day $7, \mathrm{Cl}-2$ and $\mathrm{Cl}-4$ produced mature biofilms denser than that produced by PAO1, while the biofilm formed by $\mathrm{Cl}-1$ changed very little from day $1 . \mathrm{Cl}-1$ was defective in both swarming and twitching motilities, and immunoblotting analysis confirmed that it produced a reduced level of PilA protein. The twitching-motility defect of $\mathrm{Cl}-1$ was not complemented by a plasmid carrying intact pilA. In the $48 \mathrm{~h}$ colony biofilm assay, the $\mathrm{Cl}$ varied in susceptibility to imipenem, gentamicin and piperacillin/ tazobactam. These results suggest that: (1) the isolates produced biofilms with different structures and densities from that of PAO1; (2) biofilm formation by the isolates was not influenced by either the isolation site or the QS deficiencies of the isolates; (3) the behaviour of $\mathrm{Cl}-1$ in the different biofilm systems may be due to its lack of swarming motility and type IV pilus-related twitching motility.

\section{INTRODUCTION}

Pseudomonas aeruginosa is a versatile Gram-negative pathogen that produces severe infections in immunocompromised patients, including severely burned patients, patients with cystic fibrosis (CF) and cancer patients undergoing chemotherapy (Pollack, 2000; van Delden \& Iglewski, 1998). The severity of $P$. aeruginosa infections is due to the production of different extracellular and

†Present address: Nikon Instruments, Lewisville, TX 75057, USA.

Abbreviations: $30 \mathrm{C}_{12}-\mathrm{HSL}, \mathrm{N}$-(3-oxododecanoyl) homoserine lactone; $\mathrm{C}_{4}-\mathrm{HSL}, \mathrm{N}$-butyryl homoserine lactone; $\mathrm{CF}$, cystic fibrosis; $\mathrm{Cl}$, clinical isolate(s); max. thickness, maximum thickness; OS, quorum sensing; rc, roughness coefficient; sbr, surface-to-biovolume ratio; ssc, substratum surface coverage. cell-associated virulence factors (Pollack, 2000). These factors contribute to different aspects of $P$. aeruginosa pathogenesis, including biofilm formation (Pollack, 2000; Rumbaugh et al., 2000). Under certain conditions, bacteria attach to biotic and abiotic surfaces and develop into organized communities termed biofilms (Donlan \& Costerton, 2002). During the formation of these biofilms, bacteria proliferate and produce exopolymers (extracellular matrix) and large complex structures, which protect the bacteria from the host immune response as well as against different antimicrobials (Costerton et al., 1999; Hentzer et al., 2001; Matsukawa \& Greenberg, 2004; Stewart \& Costerton, 2001). Biofilm formation is important in the establishment of $P$. aeruginosa infections on different host tissues, including the lung alveoli of the CF patient 
(Donlan \& Costerton, 2002; Prince, 2002). P. aeruginosa also forms biofilms on medical devices such as urinary catheters (Stickler, 2002). In these settings, the antibiotic resistance engendered by biofilms presents a serious challenge to the treatment of chronic $P$. aeruginosa infections (Donlan \& Costerton, 2002).

Using microscopy, in situ reporter gene analysis and 2D electrophoretic analysis, Sauer et al. (2002) have suggested that biofilm development by $P$. aeruginosa occurs in five specific stages: reversible attachment, irreversible attachment, maturation-1, maturation-2 and dispersion. Flagellar motility facilitates the reversible attachment. After the initial attachment to the surface, $P$. aeruginosa cells move along the surface by their type IV pili and proliferate into small microcolonies (Klausen et al., 2003; O'Toole \& Kolter, 1998a). The irreversible-attachment stage is characterized by the development of these cell clusters (Sauer et al., 2002). During maturation-1 and -2, the cell cluster thickness increases and reaches maximum development (Sauer et al., 2002). During the dispersion stage, bacteria actively depart the cell clusters, possibly through flagellarmediated motility (Sauer et al., 2002).

Biofilm formation by $P$. aeruginosa involves the cell-to-cell communication quorum-sensing (QS) systems. QS is a cell-density-dependent mechanism through which bacteria coordinate different activities, including bioluminescence, plasmid conjugation and the production of different virulence factors (Donlan \& Costerton, 2002; Rumbaugh et al., 2000; Venturi, 2006). P. aeruginosa possesses at least two well-defined, interrelated QS systems, las and $r h l$, that control the production of different virulence factors (Rumbaugh et al., 2000; Venturi, 2006). Each QS system consists of two components, the autoinducer synthases (LasI and RhlI, respectively) and their cognate transcriptional regulators (LasR and RhlR, respectively) (Rumbaugh et al., 2000; Venturi, 2006). LasI is the synthase for the autoinducer $\mathrm{N}$-(3-oxododecanoyl) homoserine lactone $\left(3 \mathrm{OC}_{12}-\mathrm{HSL}\right)$, while RhlI synthesizes the autoinducer $\mathrm{N}$ butyryl homoserine lactone $\left(\mathrm{C}_{4}-\mathrm{HSL}\right)$ (Rumbaugh et al., 2000; Venturi, 2006). Based on the analysis of PAO1 and its QS-defective isogenic mutants, previous studies have suggested that in $P$. aeruginosa, QS is involved in both the initiation of biofilm formation and the maturation of the biofilm (Davies et al., 1998; De Kievit et al., 2001). The las QS system appears to be important during the late but not the early stages of biofilm development (De Kievit et al., 2001). The mature architecture of a particular biofilm also appears to be dependent on the carbon source available. Biofilms formed in glucose minimal medium develop characteristic larger structures, towers and forms that resemble mushrooms, while those formed in citrate minimal medium are much flatter and more homogeneous (Davies et al., 1998; Heydorn et al., 2002; Klausen et al., 2003; Stewart et al., 1993). Recently, Shrout et al. (2006) have suggested that the nutritional requirement influences the contribution of QS to biofilm development by $P$. aeruginosa.
Many earlier $P$. aeruginosa virulence studies (both in vitro and in vivo) have utilized isogenic mutants derived from strain PAO1. Although PAO1 was originally obtained from a human infection (infected wound) (Holloway et al., 1979), it has been passaged in vitro in different laboratories for several decades. Thus, PAO1 may no longer be similar to freshly isolated $P$. aeruginosa strains. A series of $P$. aeruginosa strains isolated from CF patients had larger genome sizes and exhibited greater genomic diversity than PAO1 (Head \& Yu, 2004). Analyses of $P$. aeruginosa clinical isolates (CI) have provided further insight into the mechanisms of $P$. aeruginosa infections. Rumbaugh et al. (1999) have shown that compared to CI from respiratory tract infections, CI from urinary tract and wound infections produce more exotoxin A and exoenzyme $\mathrm{S}$, and that prolonged infection with a strain enhances exoenzyme S production. Roy-Burman et al. (2001) have shown a strong correlation between the expression of type III secretion proteins by $\mathrm{CI}$ and patient death. Head \& Yu (2004) have shown that $P$. aeruginosa isolates from CF patients differ among each other and also in comparison with non-CF isolates in many aspects of biofilm formation. In addition, Lee et al. (2005) have found differences in the ability of CF isolates to form biofilms. They also suggest that biofilm development may not be necessary for the longitudinal survival of non-mucoid $P$. aeruginosa during chronic infection of the CF lung, as the ability of sequential isolates to form biofilm in vitro decreases over time (Lee et al., 2005).

We have recently characterized five QS-deficient CI of $P$. aeruginosa. The isolates produce no LasB elastase, and variable levels of exotoxin A and exoenzyme S; they also vary in their swimming and twitching motilities (Schaber et al., 2004). Analysis of biofilm initiation using the crystal violet assay revealed that four of the isolates do not effectively adhere to the polystyrene surface (Schaber et al., 2004). In this study, we extended our biofilm analysis of these CI to include the flow-through continuous-culture system and the colony-biofilm system. In addition, we further characterized one of the isolates with respect to pilin production.

\section{METHODS}

Bacterial strains, media and growth conditions. The prototypic P. aeruginosa strain PAO1 (Holloway et al., 1979) was used as a positive control for biofilm formation and for different assays. Its isogenic pilA mutant PAO $\Delta$ pilA (Klausen et al., 2003) was used as a negative control for the analysis of twitching motility and the PilA protein. CI-1 to CI-5 were obtained from human infections: CI-1 from a wound infection, CI-2 and CI- 4 from tracheal aspirates obtained 1 month apart from a patient with lower respiratory tract infection, CI-3 from sputum of a patient with lower respiratory tract infection and CI-5 from a urinary tract infection (catheterized urine) (Schaber et al., 2004). Strains were routinely grown in Luria-Bertani (LB) broth (Miller, 1972) at $37{ }^{\circ} \mathrm{C}$ with shaking (250 r.p.m.). Biofilm formation was examined using M63 minimal medium (M63GCA) (13.6 g KH $\mathrm{KHO}_{4} \mathrm{l}^{-1}, 2.0 \mathrm{~g}\left(\mathrm{NH}_{4}\right)_{2} \mathrm{SO}_{4} \mathrm{l}^{-1}, 0.5 \mathrm{mg}$ $\mathrm{FeSO}_{4} .7 \mathrm{H}_{2} \mathrm{O}^{-1}, \mathrm{pH} 7.0$ ) supplemented with $0.2 \%$ (w/v) glucose, 
$1 \mathrm{mM} \mathrm{MgSO} 4.7 \mathrm{H}_{2} \mathrm{O}$ and $0.5 \%(\mathrm{w} / \mathrm{v})$ Casamino acids (Miller, 1972; O’Toole \& Kolter, 1998b).

Flow-through continuous culture. Development of the $P$. aeruginosa biofilm on a glass surface $\left(\sim 400 \mathrm{~mm}^{2}\right)$ was monitored using a multi-cell flow-through continuous-culture system at $37{ }^{\circ} \mathrm{C}$ (Davies et al., 1998). Polycarbonate flow devices (Protofab) were sealed with glass coverslips $(45 \times 50 \mathrm{~mm}, 1.5 \mathrm{~mm}$ thickness $)$ and secured with stainless steel brackets. The coverslips were pretreated with $0.5 \mathrm{M} \mathrm{HCl}$ for $1 \mathrm{~h}$. The flow cells were attached to \#16 silicone tubing and sterilized by autoclaving. Sterile M63GCA was maintained in a 101 reservoir and pumped to the flow cell through one-eighth inch (internal diameter) silicone tubing using a six-roller-head peristaltic pump (Masterflex). The flow rate was maintained at $0.6 \mathrm{ml} \mathrm{min}{ }^{-1}$. The fluid residual time was $\sim 3$ min with laminar flow. M63GCA that passed through the chamber was collected in a second 101 reservoir. Overnight cultures were subcultured (1:100), grown in M63GCA for $\sim 3 \mathrm{~h}$ and then diluted in fresh M63GCA to $\mathrm{OD}_{600} 0.02\left(\sim 10^{7}\right.$ c.f.u. $\mathrm{ml}^{-1}$ ) (DU-70 Spectrophotometer, Beckman). A $3 \mathrm{ml}$ aliquot of this diluted culture was injected by syringe immediately upstream of the flow cell. After inoculation, flow was stopped for $1 \mathrm{~h}$ to allow the bacteria to attach to the glass surface and then followed by continuous flow until the end of the experiment.

Microscopy of the biofilms. Syto 61 red fluorescent nucleic acid stain (Invitrogen), which stains red both live and dead cells, was used according to the manufacturer's recommendations to visualize biofilms. Biofilm formation by each strain was examined in three separate experiments. Seven image stacks were obtained from random positions within the middle section of each flow cell for a total of 21 image stacks for each strain. Images were acquired at $1-3 \mu \mathrm{m}$ intervals through the biofilms using an Olympus IX71 Fluoview 300 confocal laser scanning microscope through a UPlanApo $\times 40 / 1.00$ numerical aperture oil objective and a $633 \mathrm{~nm}$ Red Helium Neon laser (Olympus America). Biofilm image reconstruction was performed using NISElements 2.2 (Nikon Instruments).

COMSTAT analysis of biofilms. The 21 image stacks obtained per strain were analysed using the COMSTAT program (Heydorn et al., 2000). We examined the following parameters (Table 1): substratum coverage (ssc), a reflection of the efficiency with which the strain colonizes the surface; mean thickness, the mean height of the biofilm; maximum (max.) thickness; roughness coefficient (rc), a measure of how much the thickness of the biofilm varies; and surface-to-biovolume ratio (sbr), an estimate of the portion of the biofilm exposed to nutrients.

Real-time quantitative PCR. This was done as previously described (Carty et al., 2003). Briefly, overnight cultures of each strain were subcultured into fresh M63GCA and grown to $\mathrm{OD}_{600}$ 2.2-3.2. Total RNA was extracted by the hot phenol method and cDNA was synthesized using random oligonucleotides. The cDNA was then subjected to PCR using specific lasI and rhlI primers (lasI: forward 5' TTCGCCATCAACTCTGGACA-3' ${ }^{\prime}$, reverse $5^{\prime}$-CGTACAGTCGGAAAAGCCCA-3'; rhli: forward 5'-GGCGATGAAGATATTCTGGTCC3', reverse 5'-ACTGGCGCCCAGGTACC-3'). Detection of amplified products was accomplished using the ABI Prism 7000 Sequence Detection System (Applied Biosystems). Measurement of 16S rRNA was used as an internal standard. Each real-time quantitative PCR experiment was repeated twice using separate cultures. In each experiment, duplicate pellets were obtained for RNA extraction.

Swarming and twitching motilities. Swarming plates consisted of nutrient broth $\left(8 \mathrm{~g} \mathrm{l}^{-1}\right)$, glucose $\left(5 \mathrm{~g} \mathrm{l}^{-1}\right)$ and agar $(0.5 \%$, w/v) (Boles et al., 2005). The plates were dried at room temperature for several hours, inoculated with overnight cultures of the strains and incubated at $32{ }^{\circ} \mathrm{C}$ for $48 \mathrm{~h}$. Swarming motility was compared by measuring the diameters of the colonies on the plates.

Twitching motility was measured as previously described (Schaber et al., 2004; Deziel et al., 2001). Briefly, individual colonies of the strains were stab-inoculated through the agar to the bottom of $1 \%$ (w/v) agar plates. Following incubation at $32{ }^{\circ} \mathrm{C}$ for $24 \mathrm{~h}$, the agar was removed and the bacterial growth on the plastic surface was visualized with $1 \%(\mathrm{w} / \mathrm{v})$ crystal violet. Twitching motility was determined by measuring the diameter of the stained growth. Assays for both twitching and swarming motility were repeated at least three times.

Immunoblotting analysis. $P$. aeruginosa strains were grown overnight in LB broth and adjusted to equivalent $\mathrm{OD}_{600}$ with $\mathrm{LB}$ broth. Whole-cell extracts were prepared from $1 \mathrm{ml}$ cell pellets by resuspending the cells in $100 \mu$ lysis-loading buffer $(125 \mathrm{mM}$ Tris/ $\mathrm{HCl}, \mathrm{pH}$ $6.8,20 \%, \mathrm{v} / \mathrm{v}$, glycerol, $4 \%$, w/v, SDS, $0.005 \%$, w/v, bromophenol blue and $700 \mathrm{mM}$ 2-mercaptoethanol) and boiling for $5 \mathrm{~min}$. Proteins were separated by $15 \%$ SDS-PAGE and transferred to PVDF membranes (Immun-Blot, Bio-Rad). Membranes were probed for PilA using rabbit polyclonal anti-PilA antibody at a dilution of $1: 20000$. The probed membranes were treated with anti-rabbit

Table 1. Quantitative analysis of 1- and 7-day-old biofilms formed by PAO1 and the $\mathrm{Cl}$

The analysis was conducted using the COMSTAT program. In all experiments, images were acquired from random positions in the middle of the flow channel. Results represent the mean \pm SEM of three independent experiments (seven image stacks per experiment with a fixed threshold).

\begin{tabular}{|c|c|c|c|c|c|c|c|}
\hline \multirow[t]{2}{*}{ Biofilm characteristic } & \multirow[t]{2}{*}{ Day } & \multicolumn{6}{|c|}{ Strain } \\
\hline & & PAO1 & CI-1 & $\mathrm{CI}-2$ & CI-3 & CI-4 & CI-5 \\
\hline \multirow[t]{2}{*}{$\operatorname{ssc}(\%)$} & 1 & $59.02 \pm 2.55$ & $7.50 \pm 0.96$ & $6.92 \pm 1.06$ & $0.24 \pm 0.03$ & $2.78 \pm 0.41$ & $16.47 \pm 2.28$ \\
\hline & 7 & $72.29 \pm 4.73$ & $9.62 \pm 2.10$ & $77.35 \pm 5.75$ & $19.14 \pm 4.88$ & $87.44 \pm 3.74$ & $32.52 \pm 4.84$ \\
\hline \multirow[t]{2}{*}{ Mean thickness $(\mu \mathrm{m})$} & 1 & $3.09 \pm 0.23$ & $1.07 \pm 0.17$ & $0.17 \pm 0.03$ & $0.01 \pm 0.00$ & $0.07 \pm 0.01$ & $0.71 \pm 0.12$ \\
\hline & 7 & $25.25 \pm 5.33$ & $1.12 \pm 0.31$ & $8.88 \pm 1.06$ & $2.43 \pm 0.74$ & $14.21 \pm 1.02$ & $7.02 \pm 1.14$ \\
\hline \multirow[t]{2}{*}{ Max. thickness $(\mu \mathrm{m})$} & 1 & $10.62 \pm 0.22$ & $26.52 \pm 0.91$ & $7.00 \pm 0.47$ & $6.14 \pm 0.24$ & $8.00 \pm 0.33$ & $8.81 \pm 0.36$ \\
\hline & 7 & $61.67 \pm 12.44$ & $29.76 \pm 3.80$ & $20.76 \pm 1.09$ & $23.33 \pm 1.37$ & $26.52 \pm 0.98$ & $79.81 \pm 7.50$ \\
\hline \multirow[t]{2}{*}{$\mathrm{rc}$} & 1 & $0.66 \pm 0.04$ & $1.78 \pm 0.03$ & $1.87 \pm 0.02$ & $2.00 \pm 0.00$ & $1.94 \pm 0.01$ & $1.58 \pm 0.06$ \\
\hline & 7 & $0.37 \pm 0.07$ & $1.78 \pm 0.05$ & $0.47 \pm 0.12$ & $1.47 \pm 0.14$ & $0.21 \pm 0.05$ & $1.41 \pm 0.07$ \\
\hline \multirow[t]{2}{*}{$\operatorname{sbr}\left(\mu \mathrm{m}^{-2} \mu \mathrm{m}^{-3}\right)$} & 1 & $3.97 \pm 0.12$ & $3.38 \pm 0.12$ & $6.44 \pm 0.25$ & $6.91 \pm 0.30$ & $6.62 \pm 0.07$ & $5.30 \pm 0.08$ \\
\hline & 7 & $3.33 \pm 0.28$ & $6.28 \pm 0.45$ & $3.44 \pm 0.57$ & $5.30 \pm 0.40$ & $2.17 \pm 0.42$ & $3.83 \pm 0.38$ \\
\hline
\end{tabular}


horseradish peroxidase-conjugated IgG (Sigma-Aldrich) and developed using SuperSignal West Pico chemiluminescent substrate (Pierce Biotechnology). P. aeruginosa strain PAO1 was used as a positive control while its PilA-deficient isogenic mutant PAO $\Delta$ pilA (Klausen et al., 2003) was used as a negative control.

Phage adsorption (efficiency of plating). Phage F116L was amplified by standard methods (Kutter \& Sulakvelidze, 2005; Martin et al., 1993) and serially diluted in 10-fold steps. Each tested bacterial strain was grown to $\mathrm{OD}_{600} 0.5\left(\sim 10^{9}\right.$ c.f.u. $\left.\mathrm{ml}^{-1}\right)$ in $\mathrm{LB}$ broth. A $100 \mu \mathrm{l}$ aliquot of the culture was incubated with $100 \mu \mathrm{l}$ of each F116L phage dilution for $10 \mathrm{~min}$ at $37{ }^{\circ} \mathrm{C}$. Each mixture was added to $3 \mathrm{ml} \mathrm{LB}$ soft agar $\left(7.5 \mathrm{~g} \mathrm{l}^{-1}\right)$ and the soft agar was poured over LB agar plates. The plates were incubated overnight at $37{ }^{\circ} \mathrm{C}$. Efficiency of plating was calculated as mean phage titre determined for each bacterial strain divided by the mean phage titre determined for PAO1 (Kutter \& Sulakvelidze, 2005). Each experiment was performed in triplicate.

Colony biofilm assay. These experiments were conducted using 25 $\mathrm{mm}$ diameter, $0.22 \mu \mathrm{m}$ pore-size black polycarbonate membrane filters (Poretics), as described elsewhere (Anderl et al., 2003). Both sides of the membrane filters were exposed to UV light for $15 \mathrm{~min}$ and the membranes were gently pressed onto the surface of M63GCA agar $(1.8 \%, \mathrm{w} / \mathrm{v})$ plates. $P$. aeruginosa strains grown overnight in LB broth at $37^{\circ} \mathrm{C}$ were subcultured $1: 100$ in M63GCA and incubated at $37^{\circ} \mathrm{C}$ for $3 \mathrm{~h}$. The subcultures were diluted in fresh M63GCA to $\mathrm{OD}_{600} 0.2$ $\left(\sim 10^{7}\right.$ c.f.u. $\left.\mathrm{ml}^{-1}\right)$ and $10 \mu \mathrm{l}$ of each diluted strain was spotted in the centre of the membrane on the M63GCA agar plate and allowed to dry. Inverted plates were incubated at $37{ }^{\circ} \mathrm{C}$ for a total of $48 \mathrm{~h}$, and the membranes were transferred to fresh M63GCA agar plates at $24 \mathrm{~h}$. Colony biofilms were harvested by aseptically transferring the membranes to $10 \mathrm{ml}$ sterile PBS (pH 7.0) and vortexing for $2 \mathrm{~min}$. The cell suspensions were serially diluted in PBS and drop-plated (10 $\mu \mathrm{l}$ aliquots) on LB agar. Plates were allowed to dry, inverted and incubated at $37{ }^{\circ} \mathrm{C}$ overnight. Numbers of micro-organisms were calculated as c.f.u. per square centimetre of the membrane.

Antibiotic susceptibility of the colony biofilms. This was determined by transferring the $48 \mathrm{~h}$ biofilms from the M63GCA agar plates onto LB plates supplemented with at least ten times the MIC of antibiotic previously determined for planktonic cells of CI-1 and PAO1 (data not shown; Schaber et al., 2004); that is, imipenem at 40 $\mu \mathrm{g} \mathrm{ml}^{-1}$, gentamicin at $40 \mu \mathrm{g} \mathrm{ml}^{-1}$ and piperacillin/tazobactam at 80 $\mu \mathrm{g} \mathrm{ml}^{-1}$. After $16 \mathrm{~h}$ incubation at $37^{\circ} \mathrm{C}$, the membranes were lifted from the antibiotic agar plates and c.f.u. were determined as described above. Killing of the biofilm cells was calculated as log reduction according to the following formula (Anderl et al., 2003):

$-\log _{10}$ (c.f.u. with antibiotic/c.f.u. without antibiotic).

Significant differences in log reductions between PAO1 and CI-1 were calculated by unpaired $t$ test using GraphPad InStat version 3.00 for Windows 95 (GraphPad Software).

\section{RESULTS}

\section{Biofilm formation by the QS-deficient $\mathrm{Cl}$}

We have previously described five $\mathrm{CI}$ that are defective in QS, producing $2-20 \%$ of the $3 \mathrm{OC}_{12}-\mathrm{HSL}$ elaborated by PAO1 and $0-34 \%$ of the $\mathrm{C}_{4}$-HSL (Schaber et al., 2004). Using the crystal violet biofilm assay, we showed that four of the five CI were less than $35 \%$ as efficient as PAO1 in the initiation of biofilms, while one was $82 \%$ as efficient (Schaber et al., 2004).
The QS defects in these CI may also influence their ability to develop a mature biofilm. Therefore, to determine if one or more was able to form mature biofilms and to examine the structure of these biofilms, we monitored biofilm formation by the CI using a flow-through continuous-culture system irrigated with M63 minimal medium containing glucose as a carbon source (M63GCA) (O'Toole \& Kolter, 1998b). As a control, we utilized the $P$. aeruginosa strain PAO1, which forms a well-developed characteristic mature biofilm in this medium (O'Toole \& Kolter, 1998b). Using confocal laser scanning microscopy, we observed biofilm initiation at day 1 post-inoculation and maturation of the biofilm at day 7 . We also analysed the biofilm quantitatively using the COMSTAT program (Heydorn et al., 2000).

As shown in Fig. 1 and Table 1 , and briefly described below, the day 1 and day 7 biofilms formed by the CI differed from that produced by PAO1 and from each other.

PA01. The day 1 biofilm was characterized by the formation of a monolayer that covered much of the substratum surface $(59 \%)$ with multiple small microcolonies (rc 0.66). At day 7, PAO1 formed a more structured biofilm with regular clusters of large, differentiated microcolonies (ssc 72\%, max. thickness $61.67 \mu \mathrm{m}$, rc 0.37 ).

Cl-1. The day 1 biofilm was significantly less dense than that of PAO1 (ssc $7.5 \%$ ), and the microcolonies were fewer but larger than those of the PAO1 day 1 biofilm (max. thickness $26.52 \mu \mathrm{m}$ compared to $10.62 \mu \mathrm{m}$ ). In the day 7 biofilm, the glass surface was evenly spotted with these microcolonies (ssc 9.6\%), but they had changed little in height (max. thickness $29.8 \mu \mathrm{m}$ ). At both day 1 and day 7 , this CI produced the most variable biofilm of all isolates tested ( $\mathrm{rc}$ on both days of 1.78).

Cl-2. The day 1 biofilm showed a less dense ssc than PAO1 $(7 \%)$ with small microcolonies visible (max. thickness $7 \mu \mathrm{m})$. However, by day 7 the surface was more densely covered with a rougher biofilm than PAO1 (ssc $77 \%$, rc 0.5 compared to 0.37). In addition, large filaments were observed arching among the microcolonies (data not shown).

Cl-3. At day 1 , very few bacteria had attached to the surface (ssc $0.2 \%$ ), forming sparse microcolonies with a max. thickness of only $6.14 \mu \mathrm{m}$, but by day 7 , the strain covered $19 \%$ of the glass surface with microcolonies reaching $23.33 \mu \mathrm{m}$ in height.

Cl-4. This strain was a sequential isolate from the same patient and same site as CI-2. Its day 1 biofilm covered less surface area $(2.8 \%)$ than that of CI-2, but the day 7 biofilm covered more surface area $(87 \%)$. Its day 7 biofilm was thicker (max. thickness $26 \mu \mathrm{m}$ versus $21 \mu \mathrm{m}$ ) but smoother (rc 0.2 compared to 0.5) than that of CI-2. The same arching filaments were observed (data not shown).

Cl-5. At day 1 , this strain covered the greatest surface area of any of the CI (16\%). At day 7, $32 \%$ of the surface was 


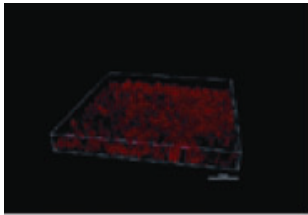

PAO1
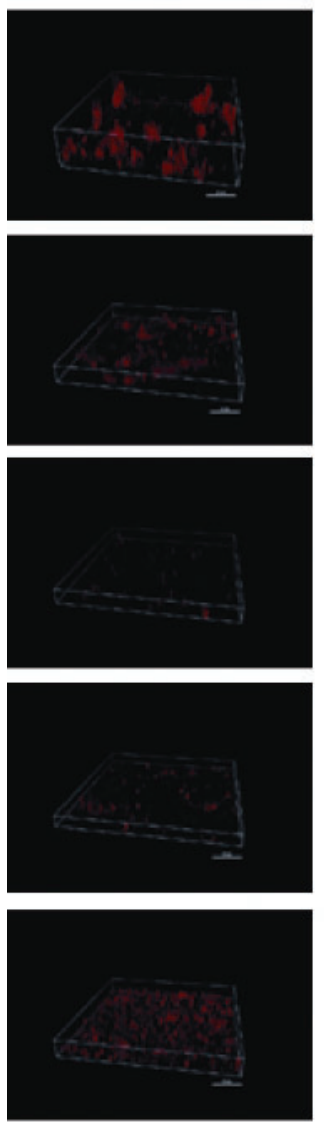

Day 1

$\mathrm{Cl}-1$

$\mathrm{Cl}-2$

Cl-3

$\mathrm{Cl}-4$

Cl-5
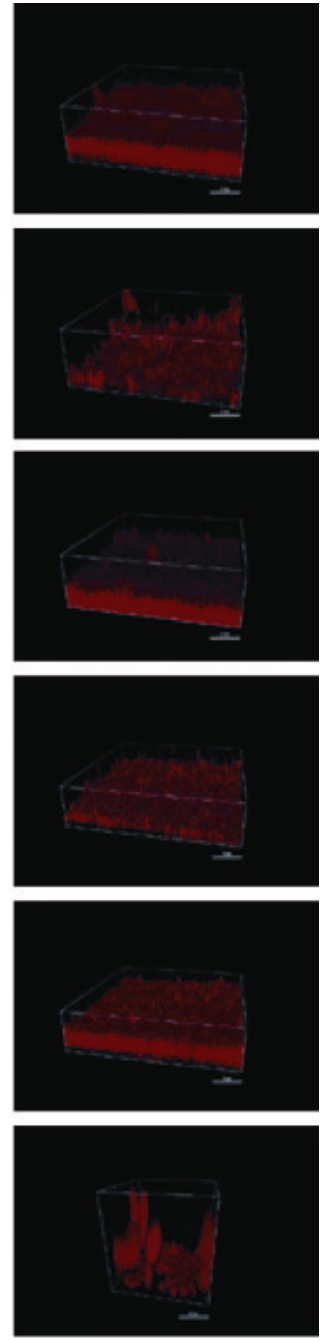

Day 7

Fig. 1. Representative structures of the biofilms formed by PAO1 and the $\mathrm{Cl}$. The strains were inoculated in flow-through continuous-culture chambers in M63GCA. The biofilms were analysed by confocal laser scanning microscopy at days 1 and 7 postinoculation. Magnification $\times 400$; bars, $20 \mu \mathrm{m}$.

covered with variably sized microcolonies, including thick towers of cells reaching $80 \mu \mathrm{m}$ in height.

These data indicate the following features of the CI biofilms. In comparison with PAO1, all CI initiated biofilm formation significantly less efficiently (ssc $0.24-16.47 \%$ ). The day $1 \mathrm{CI}$ biofilms also showed higher degrees of diversity (all rc greater than that of PAO1), and except for CI-1, which was similar to PAO1, the CI produced more scattered microcolonies (sbr greater than that of PAO1). By day 7 , the surface coverage of the mature biofilms of CI-2 and CI- 4 exceeded that of PAO1, but similar to PAO1, CI-2 and CI-4 formed relatively homogeneous biofilms ( $\mathrm{rc} 0.37$, 0.47 and 0.21 ; sbr $3.33,3.44$ and 2.17 , respectively). In contrast, CI- 1 produced the least dense (scc $<10 \%$ of that

of PAO1) and most heterogeneous ( $\mathrm{rc} 1.78$, sbr 6.28) mature biofilm of the CI. The mature biofilm of CI-3 was also less dense and more heterogeneous than that of PAO1 (scc $19 \%$, rc 1.47, sbr 5.3). CI-5, which formed a mature biofilm with moderate ssc and heterogeneity, produced unique towering structures, yet its sbr was similar to that of PAO1 (3.82 versus 3.33). Next, we compared the general growth characteristics of planktonic cells of the CI with those of PAO1. As shown in Fig. 2, there were no major differences in the growth characteristics of the CI and $\mathrm{PAO} 1$ at the early exponential, mid-exponential or stationary phases of growth.

\section{lasl and rhll transcription in the $\mathrm{Cl}$}

Using the flow-through continuous-culture system, Davies et al. (1998) suggested that the lasI gene is important in the development of PAO1 biofilm. In addition, De Kievit et al. (2001) showed that during the course of an 8 day biofilm, lasI expression decreases progressively, while that of rhlI is steady but occurs in a low percentage of cells. With the exception of CI-5, our isolates produced significantly lower levels of $3 \mathrm{OC}_{12}-\mathrm{HSL}$ and $\mathrm{C}_{4}$-HSL, yet PCR analysis of their chromosomes suggested that all the $\mathrm{CI}$ carried the lasI and rhlI genes that code for the $3 \mathrm{OC}_{12}-\mathrm{HSL}$ and $\mathrm{C}_{4}-\mathrm{HSL}$ synthases, respectively (Schaber et al., 2004). Therefore, we used quantitative real-time PCR to determine whether lasI and rhlI were transcribed in the $\mathrm{CI}$ as well as the relative levels of any transcription. As shown in Fig. 3, the levels of lasI transcripts produced by the isolates ranged from 37$71 \%$ of that produced by PAO1. Similarly, the levels of rhlI transcripts ranged from $35-92 \%$ of that produced by PAO1 (Fig. 3). This suggests that the defect in the production of $3 \mathrm{OC}_{12}-\mathrm{HSL}$ and $\mathrm{C}_{4}-\mathrm{HSL}$ is not due to inefficient expression of the lasI or rhlI genes. In addition, despite the lack of functional lasR or rhlR genes (Schaber et al., 2004), CI-2 and CI-4 still developed mature biofilms (Fig. 1, Table 1).

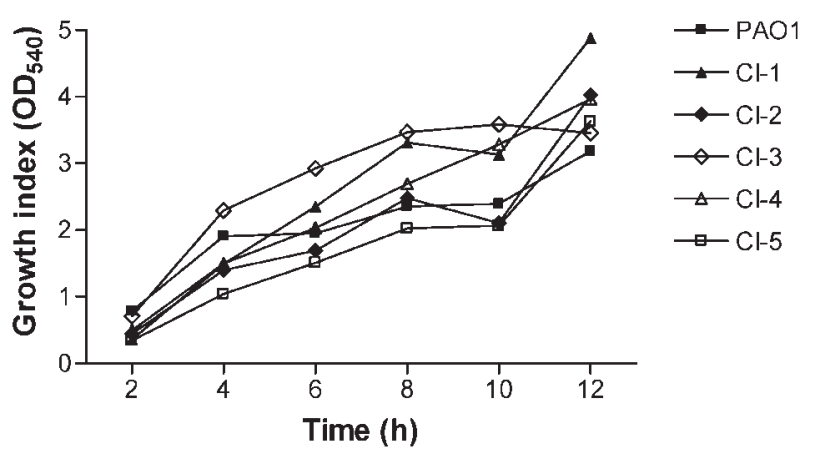

Fig. 2. Comparison of the growth rates of $P A O 1$ and the $\mathrm{Cl}$. An overnight culture of each strain was inoculated into fresh LB broth to $\mathrm{OD}_{540} 0.03-0.05$. Cells were grown at $37{ }^{\circ} \mathrm{C}$ with shaking for $12 \mathrm{~h}$. Samples were obtained at the indicated times and the $\mathrm{OD}_{540}$ (indicative of growth rate) of each sample was determined. 


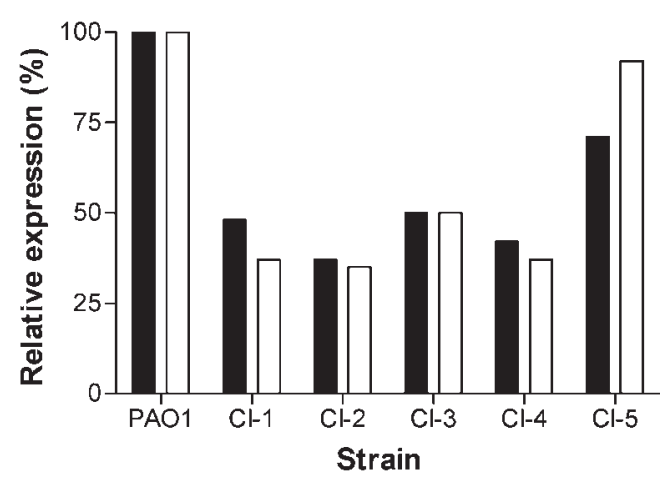

Fig. 3. Relative expression of las/ and $r h / l$ in PAO1 and different $\mathrm{Cl}$, as determined by real-time quantitative PCR. Cells were grown in M63GCA to $\mathrm{OD}_{600}$ 2.2-3.2. RNA extraction and PCR experiments were conducted as previously described (Carty et al., 2003). Relative expression of las/ (black bars) and rhll (white bars) in the $\mathrm{Cl}$ is reported as a percentage of that detected in PAO1 (the level of expression in PAO1 was taken as $100 \%$ ). Each real-time PCR experiment was repeated twice using separate cultures. In each experiment, duplicate pellets were obtained for RNA extraction.

\section{Examining the $\mathrm{Cl}$ for swarming and twitching motilities}

Shrout et al. (2006) have recently shown that the carbon source plays an important role in the ability of $P$. aeruginosa QS mutants to develop a biofilm. This effect appears to occur through the regulation of swarming motility by the QS systems (Shrout et al., 2006). Therefore, we examined the $\mathrm{CI}$ for their swarming motility. As shown in Fig. 4, CI-2 and CI-4 swarmed better than the PAO1 control strain, while CI-5 swarmed less then PAO1. CI-3 showed a considerable reduction in its swarming motility,

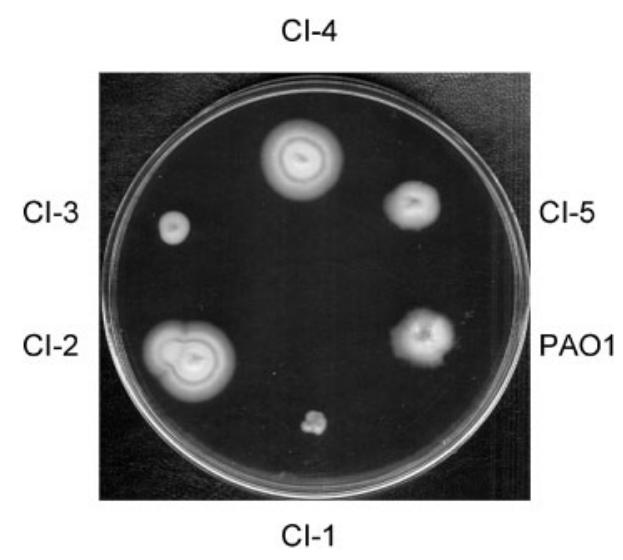

Fig. 4. Swarming motility of $\mathrm{PAO} 1$ and the $\mathrm{Cl}$. Swarming motility was assayed on nutrient agar swarming plates containing $5 \mathrm{~g}$ glucose $\mathrm{I}^{-1}$ and $0.5 \%(\mathrm{w} / \mathrm{v})$ agar incubated at $32{ }^{\circ} \mathrm{C}$ for $48 \mathrm{~h}$. The assay for swarming motility was repeated at least three times. while CI-1 did not swarm (Fig. 4). We have previously reported that the CI also produce variable levels of twitching motility (Schaber et al., 2004). However, in that study we did not compare the isolates with a twitchingmotility-deficient mutant of PAO1 (a negative control). Thus, in this study we re-examined the CI for twitching motility in comparison with PAO $\Delta$ pilA using the twitching-motility plate assay (Deziel et al., 2001). Compared to PAO1, which produced the typical twitching-motility phenotype, neither PAO $\Delta$ pilA nor CI-1 produced distinctive twitching motility (Fig. 5a). The rest of the CI were not defective in their twitching motility (data not shown).

CI-1 produced the least dense and most heterogeneous biofilm of the CI (Fig. 1, Table 1). Klausen et al. (2003) have suggested that after the initial phase of biofilm formation, the type IV pilus (through its contribution to twitching motility) is required for the spread of $P$. aeruginosa on the substratum. In comparison with its parent strain, the pilin-deficient mutant $\mathrm{PAO} \Delta$ pilA formed a heterogeneous biofilm with irregular structure and failed to spread on the glass surface (Klausen et al., 2003). Many features of the CI-1 mature biofilm resembled those of the biofilm formed by the type IV pilus-deficient mutant PAO $\Delta$ pilA (Fig. 1, Table 1; data not shown). To determine if the twitching-motility-negative phenotype of CI-1 is due to the loss of type IV pilin protein PilA, we examined CI-1 in immunoblotting experiments using a PilA polyclonal antibody. The $15 \mathrm{kDa}$ PilA protein was detected within the whole-cell lysate of PAO1 but not PAO $\Delta$ pilA (Fig. 5b). The whole-cell lysate of CI-1 produced a faint band that migrated at the same distance as the pilin protein of PAO1 (Fig. 5b), indicating that CI-1 produces a reduced amount of PilA. To determine whether this reduced amount of PilA leads to surface expression of the type IV pilus, we examined the ability of phage F116L (Pemberton, 1973) to infect CI-1. Phage-adsorption experiments were conducted as described in Methods. Strain PAO $\Delta$ pilA was utilized as a negative control. The efficiencies of plating for PAO1, $\mathrm{PAO} \Delta$ pilA and CI-1 were 1,0 and $1.8 \times 10^{-7}$, respectively (data not shown). These results suggest that despite the presence of low levels of PilA, CI-1 lacks twitching motility because it fails to assemble type IV pili.

To determine whether the biofilm produced by CI- 1 is indeed due to the deficiency in production of the type IV pilus, we attempted to complement the defect with intact pilA. A $776 \mathrm{bp}$ fragment that carries intact pilA (450 bp coding sequence plus upstream and downstream regions) was amplified from the chromosome of PAO1 by PCR. The fragment was cloned into the EcoRI/SacI sites of the broadhost-range cloning vector pUCP18 (Schweizer, 1991). The resulting plasmid p8566 was introduced into PAO $\Delta$ pilA and CI-1 by electroporation (Smith \& Iglewski, 1989). As shown in Fig. 5(c), p8566 complemented the defect in twitching motility of PAO $\Delta$ pilA but not CI-1. Thus, CI-1 may carry a mutation in a gene(s) that regulates the synthesis of the type IV pilus. Taken together, these results suggest that the biofilm phenotype of CI-1 may be due to 
(a)

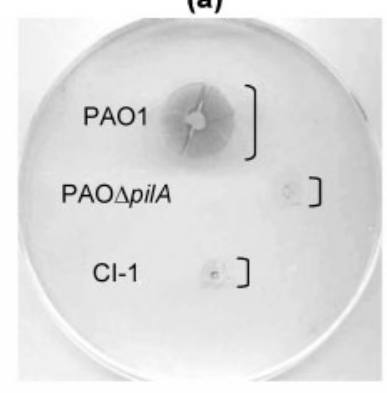

(b)

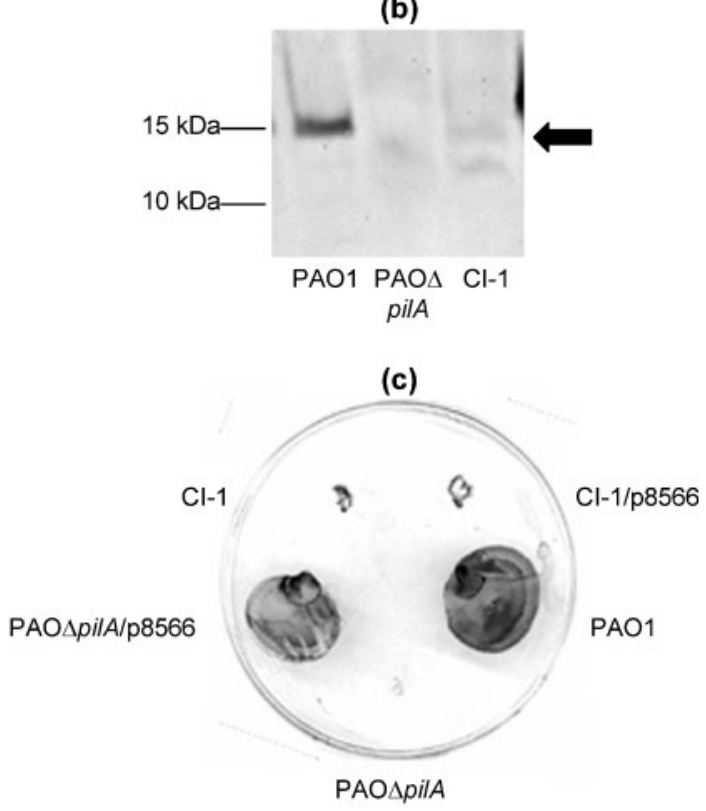

Fig. 5. Twitching motility and PilA production by PAO1, $\mathrm{PAO} \Delta$ pilA and $\mathrm{Cl}-1$. (a) Twitching motility of the strains was determined by stab inoculation of single colonies from overnight growth on LB agar into soft LB agar plates. Plates were incubated at $32{ }^{\circ} \mathrm{C}$ for $24 \mathrm{~h}$ followed by an additional $48 \mathrm{~h}$ at room temperature. Motility was evaluated by crystal violet $(1 \%, \mathrm{w} / \mathrm{v})$ staining of the growth on the plastic Petri plate under the agar. (b) Immunoblot of whole-cell lysates of PAO1, PAO $\Delta$ pilA and $\mathrm{Cl}-1$ for PilA production, as described in Methods. (c) Complementation analysis of the twitching-motility defect of PAO $\triangle$ pilA and $\mathrm{Cl}-1$ using plasmid p8566, which carries intact pilA from PAO1. Twitching motility was analysed as described in (a). The assays for twitching motility were repeated at least three times.

the deficiency of this isolate in both swarming and twitching motility.

\section{Colony biofilm formation by $\mathbf{C l}-1$}

Depending on the type of infection and the tissue to which it is attached, $P$. aeruginosa may form different biofilms. For example, CI-1 was isolated from a wound infection
(Schaber et al., 2004). It has been hypothesized that $P$. aeruginosa may form a biofilm during chronic wound infections (Costerton et al., 1999), although such a biofilm would not be exposed to fluid-flow shear forces as in a urinary tract infection (Nicolle, 2005). The colony biofilm system (Borriello et al., 2004; Walters et al., 2003) resembles the infectious environment of a wound and can be utilized to examine biofilms in vitro. Thus, we examined the $\mathrm{CI}$ in colony biofilm experiments using polycarbonate membranes, as described elsewhere (Borriello et al., 2004; Walters et al., 2003). At 48 h postinoculation, the c.f.u. $\mathrm{cm}^{-2}$ of the CI were five- to 10 -fold less than that of PAO1 $\left(1 \times 10^{6}\right.$ versus $\left.1 \times 10^{7}\right)$ (data not shown). The c.f.u. $\mathrm{cm}^{-2}$ of CI-1 was 10 -fold less than that of PAO1, which suggests that, as in the flow-through continuous-culture system, CI-1 is not as efficient as PAO1 at forming a colony biofilm.

We then examined the susceptibility of colony biofilms formed by the CI to several antibiotics. We had previously determined that the planktonic cells of the CI varied in their susceptibility to three antibiotics frequently used to treat $P$. aeruginosa infections: imipenem (carbapenem $\beta$ lactam), gentamicin (aminoglycoside) and piperacillin/ tazobactam (antipseudomonal $\beta$-lactam plus $\beta$-lactamase inhibitor) (Schaber et al., 2004). All CI except CI-3 were susceptible to imipenem and all CI except CI-5 were susceptible to piperacillin/tazobactam, while only CI-1 was susceptible to gentamicin (Schaber et al., 2004). Planktonic cells of the PAO1 strain utilized in the present study were susceptible to all three antibiotics at $\leqslant 4 \mu \mathrm{g}$ imipenem $\mathrm{ml}^{-1}, 4 \mu \mathrm{g}$ gentamicin $\mathrm{ml}^{-1}$ and $\leqslant 8 \mu \mathrm{g}$ piperacillin/ tazobactam $\mathrm{ml}^{-1}$. However, within a biofilm, the resistance to antibiotics may reach more than 10 times that of planktonic cells (Borriello et al., 2004). Therefore, we compared the antibiotic resistance of the $\mathrm{CI}$ within a colony biofilm to that of PAO1 using imipenem, gentamicin and piperacillin/tazobactam concentrations at least 10-fold higher than those for the planktonic cells: 40 , 40 (40-fold higher for CI-1) and $80 \mu \mathrm{g} \mathrm{ml}^{-1}$, respectively. Since CI-4 represents a repeated isolation of CI-2 within a 1 month period and both isolates were susceptible to all three antibiotics, we examined the antibiotic susceptibility of CI-2 colony biofilm as representative of both isolates. As shown in Fig. 6(a), the CI colony biofilms were more susceptible to imipenem than that of PAO1 (the log reductions were greater than that of PAO1), although only CI-2 and CI-3 colony biofilms were significantly more susceptible $(P<0.01)$. With respect to gentamicin, colony biofilms formed by CI- 1 and CI-5 were significantly more resistant $(P<0.001$ and 0.01 , respectively) than that of $\mathrm{PAO} 1$, while that of $\mathrm{CI}-3$ was significantly more susceptible $(P<0.001)$ (Fig. 6b). Colony biofilms formed by CI-2 and CI-3 were significantly more susceptible $(P<0.01)$ to piperacillin/tazobactam than that formed by PAO1 (Fig. 6c). It is important to note that the susceptibilities of the CI in the planktonic setting did not always correlate with their susceptibility within the biofilm. 

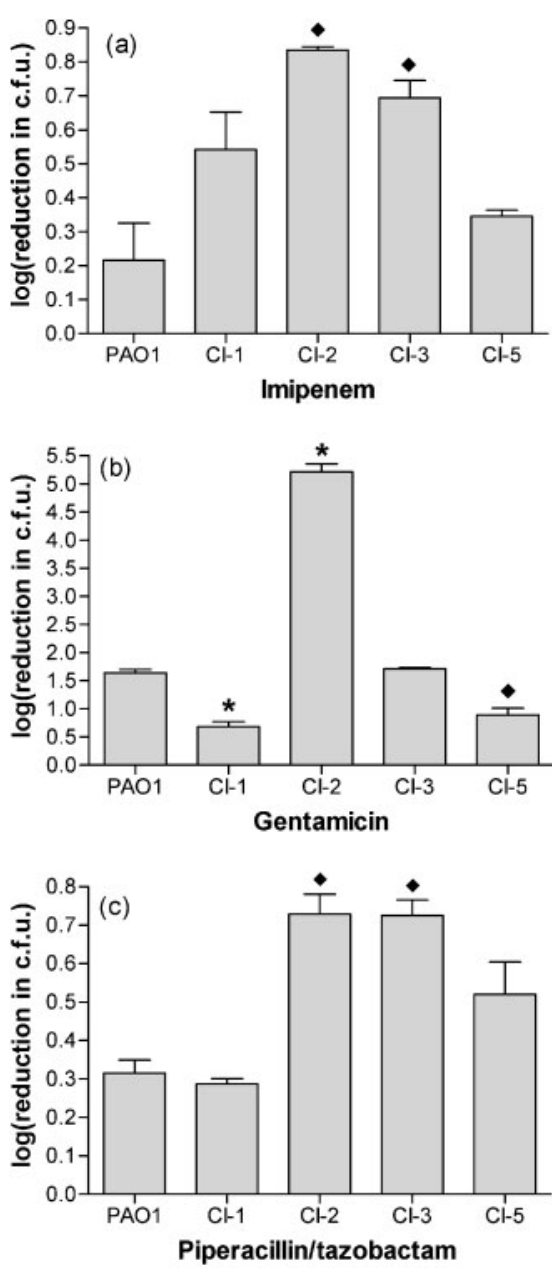

Fig. 6. Susceptibility of $\mathrm{PAO} 1$ and $\mathrm{Cl}$ biofilms to antibiotics. Strains were grown on polycarbonate membranes placed on M63GCA agar plates, as described in Methods. After $48 \mathrm{~h}$ growth, the membranes were transferred to LB agar containing (a) $40 \mu \mathrm{g}$ imipenem $\mathrm{ml}^{-1}$, (b) $40 \mu \mathrm{g}$ gentamicin $\mathrm{ml}^{-1}$ and (c) $80 \mu \mathrm{g}$ piperacillin/tazobactam $\mathrm{ml}^{-1}$. Following $16 \mathrm{~h}$ exposure to the antibiotic agar, the bacteria on the membranes were resuspended in sterile PBS, diluted and plated to determine c.f.u. Values represent the mean \pm SEM of three independent experiments. *, $P<0.001 ;, P<0.01$.

\section{DISCUSSION}

The three $P$. aeruginosa factors that are considered important for biofilm formation are flagellar-mediated swimming motility, pilus-mediated twitching motility and QS. Swimming motility is required for the initial reversible attachment stage of biofilm development. Although our five $\mathrm{CI}$ varied in the levels of their swimming motility, none was non-motile, indicating that all are flagellated (Schaber et al., 2004). However, with the exception of CI-3, there was no consistent correlation between the level of swimming motility and the initiation and early development of biofilms (1-day-old biofilm). The swimming motility of CI-3 was $23 \%$ that of PAO1 and its ability to initiate biofilm (by the crystal violet assay) was $29 \%$ that of PAO1 (Schaber et al., 2004). However, in the flow-through continuous-culture system, CI-3 produced an ssc of only $0.24 \%$, which is $0.4 \%$ of the ssc of PAO1 on day 1 (Table 1). On the other hand, the swimming motility of CI-2 and CI-4 was 92 and $100 \%$, respectively, compared to PAO1, which indicates the presence of a fully functional flagellum, yet their ability to initiate biofilm formation was 20 and $33 \%$, respectively (Schaber et al., 2004). In the flowthrough system these isolates produced an ssc at day 1 of only $7.5 \%$ (11.7\% that of PAO1) and $2.78 \%$ (4.7\% that of PAO1), respectively (Table 1). Thus, despite the presence of a fully functional flagellum, CI-2 and CI-4 showed deficiencies in the early development of their biofilms. It is possible that CI-2 and CI-4 lack an additional factor besides swimming motility that is important for biofilm initiation. However, such a defect does not appear to interfere with the ability of either strain to form mature, differentiated biofilms (Fig. 1, Table 1).

Pilus-dependent twitching motility is required for the spread of $P$. aeruginosa after its initial attachment to the surface (irreversible attachment and microcolony formation). Our results showed a good correlation between twitching motility and the efficient spread of the CI within the 7-day-old mature biofilm, with the exception of CI-5. The least efficient in its spread was CI-1 (ssc 9.62\%, or $13.3 \%$ that of PAO1) (Table 1). CI-1 was also 10-fold less efficient than PAO1 in forming a colony biofilm. Although we have previously reported that the twitching motility of CI-1 is $30 \%$ that of PAO1 (Schaber et al., 2004), our current analysis using a pilA-deficient mutant of PAO1 as a negative control revealed a considerable reduction in CI-1 twitching motility (Fig. 5a). This reduction is due to the reduced production of the PilA protein (Fig. 5b). We tried to confirm the correlation between the biofilm phenotype of CI-1 and its lack of twitching motility using complementation analysis. However, plasmid p8566, which carries the intact pilA gene, failed to complement the defect in twitching motility in CI-1 (Fig. 5c). Another possible cause of the inefficient spread of the mature CI-1 biofilm is its lack of swarming motility. As shown in Fig. 4, CI-1 is the only isolate that did not swarm. A recent study by Shrout et al. (2006) has suggested that $P$. aeruginosa swarming motility may influence the early stages of biofilm development, while Kohler et al. (2000) have suggested that QS, the flagellum and the type IV pili contribute to the effect of swarming motility on biofilm development.

Similar to CI-1, the reduced twitching motility of CI-3 correlated with its inefficient spread within the mature biofilm (ssc $19.14 \%$, or $26.5 \%$ that of PAO1) (Table 1). In contrast, both CI-2 and CI-4 showed no reduction in either their twitching motility (100 and $92 \%$ of PAO1, respectively) (Schaber et al., 2004) or their spread within the mature biofilm (ssc 77.35 and $87.44 \%$, or 107 and $121 \%$ that of PAO1) (Table 1). Despite the apparent uncompromised twitching motility of CI-5 (92\% that of PAO1) (Schaber et al., 2004), its efficiency of spread within the 
mature biofilm was about half that of PAO1 (ssc $32.52 \%$, or $44.9 \%$ that of PAO1) (Table 1). Unlike the other CI, CI5 produced a mature biofilm with unique features: unusually tall cell clusters (Fig. 1). Whether the twitching motility contributed to this unique feature is not known at this time. Since CI-4 had similar levels of twitching motility yet covered more substratum surface than PAO1, this suggests that there is a possible defect in the pili of CI-5, or that some factor other than twitching motility is involved in its unique architecture.

The effect of the QS systems on the different virulence attributes of $P$. aeruginosa has been documented (Davies et al., 1998; De Kievit et al., 2001). The findings of earlier reports vary with respect to the role of QS in biofilm formation by $P$. aeruginosa. Using a glass surface as a substratum and QS isogenic mutants of PAO1, Davies et al. (1998) showed that in comparison with PAO1, a lasI mutant produces a thin biofilm that is easily dispersed when treated with a detergent such as SDS. Using similar conditions, De Kievit et al. (2001) showed that during the course of an 8-day biofilm, lasI expression decreases progressively, while that of rhlI is steady but occurs in a low percentage of cells. Our analysis of the CI suggests that QS may not play a major role in biofilm initiation and the spread of $P$. aeruginosa over the substratum. We have previously demonstrated that with the exception of CI-5, the CI produce significantly lower levels of $3 \mathrm{OC}_{12}-\mathrm{HSL}$ and no detectable $\mathrm{C}_{4}$-HSL (Schaber et al., 2004). In addition, the CI produce considerably lower levels of the QScontrolled factors LasB and pyocyanin (Schaber et al., 2004). Despite that, the CI produced mature biofilms of variable density (Fig. 1, Table 1). Our analysis also suggests that certain components of the QS systems (lasI, rhlI, lasR and $r h l R$ ) play a role in the development of certain features of the mature biofilms. We previously showed that CI-2 and CI-4 carry deletions within the lasR and rhlR genes (Schaber et al., 2004). In addition, neither CI-2 nor CI-4 produced detectable lasR or $r h l R$ transcripts (data not shown). However, CI-2 and CI-4 formed mature biofilms that covered the glass surface more densely than that of PAO1, while those formed by CI- $1, \mathrm{CI}-3$ and CI- 5 were less dense (Fig. 1, Table 1). In addition, the biofilms produced by CI-1, CI-3 and CI-5 were more heterogeneous than those of PAO1, while that of CI-4 was less heterogeneous (Fig. 1, Table 1). Thus, lasR and rhlR may contribute to the heterogeneity of the biofilms.

The detection of lasI and rhlI transcripts in CI-2 and CI-4 (about $40 \%$ that of PAO1) (Fig. 3) is puzzling. We previously indicated that CI-2 and CI-4 carry deletions in both lasR and rhlR (Schaber et al., 2004). Using PCR analysis, we failed to amplify DNA fragments (either internal fragments or fragments that carry the intact genes) from the chromosomes of CI-2 and CI-4 (Schaber et al., 2004). In addition, we showed that both $\mathrm{CI}-2$ and $\mathrm{CI}-4$ produce significantly reduced levels of $3 \mathrm{OC}_{12}-\mathrm{HSL}$ and $\mathrm{C}_{4}$-HSL in comparison (Schaber et al., 2004). We had not expected to detect lasI and rhlI mRNA, as $3 \mathrm{OC}_{12}$-HSL-activated LasR and $\mathrm{C}_{4}$-HSL-activated RhlR induce expression of lasI and rhlI, respectively (Venturi, 2006). The apparent discrepancy between the present results and those of earlier studies (Latifi et al., 1996; Seed et al., 1995) may be due to differences in the sensitivity of the assays and the parameters that were examined in each assay. The previous studies utilized detection of $\beta$-galactosidase activity (lac $Z$ fusion system), which determines the efficiency of lasI and rhlI expression from their promoters, while we utilized more sensitive real-time PCR that measures the amount of accumulated lasI and rhlI mRNA. Alternatively, other pathways may exist whereby $P$. aeruginosa obtains lasI and rhlI transcripts in the absence of autoinducer-activated LasR and RhlR. For example, Carty et al. (2006) have recently suggested that the $P$. aeruginosa regulatory protein PtxR enhances transcription of lasI but does not affect lasR.

Using PAO1 and its QS-isogenic mutants, Shrout et al. (2006) have recently suggested that the contribution of QS to the development of a PAO1 biofilm depends on the growth medium, specifically the carbon source. In the presence of glucose in the biofilm medium, both PAO1 and its QS mutants produce thin monolayers with some cell aggregates, and PAO1 and its PAO1 QS mutants do not swarm on their glucose medium (Shrout et al., 2006). In the presence of succinate, PAO1, which is able to swarm, produces a flat uniform biofilm, while the QS-mutants produce cell aggregates (Shrout et al., 2006). In the present study, we utilized glucose as a carbon source in our biofilm medium also (see Methods), in which PAO1, CI-2 and CI4 produced flat uniform mature biofilms that covered the substratum (Fig. 1, Table 1). In addition, we did not find PAO1 to be defective in its swarming motility (Fig. 4). We suggest three reasons for the observed differences between the two studies. First, different media were utilized to examine biofilm development and swarming motility. While Shrout et al. (2006) utilized modified FAB medium that contained either succinate or glucose as a carbon source, we utilized M63 minimal medium that was supplemented with glucose and Casamino acids (O'Toole \& Kolter, 1998b). The swarming plates described by Shrout et al. (2006) consisted of FAB medium that was supplemented with either succinate or glucose. Our swarming plates contained glucose and nutrient broth (Boles et al., 2005). Second, Shrout et al. (2006) examined biofilm development primarily within $48 \mathrm{~h}$ post-inoculation, while we examined the mature biofilm 7 days post-inoculation. Third, Shrout et al. (2006) utilized PAO1 isogenic mutants that were defective in either lasI/rhlI or lasR/rhlR, while our QS-deficient CI were genotypically different from PAO1.

Comparison of our results with those of Lee et al. (2005) provided the following observations. (1) According to Lee et al. (2005), twitching motility affects the architecture of the $P$. aeruginosa biofilms. Isolates that retain twitching motility produce flat, homogeneous biofilms while those lacking twitching motility produce heterogeneous biofilms with irregular microcolonies. Similar to the findings of Lee et al. (2005), CI-1, which is twitching-motility deficient, 
produced a heterogeneous biofilm with irregular microcolonies (Fig. 1, Table 1). (2) In the Lee et al. (2005) study, the effect of QS on biofilm formation was not clear, since isolates that failed to produce either one or both autoinducers also lacked twitching motility. One isolate that was obtained six times from a single patient over a 23 year period showed variations in biofilm development during sequential isolation. The first three sequential isolates (positive for twitching motility and both autoinducers) formed monolayers within the first $24 \mathrm{~h}$ and a flat biofilm structure that covered the substratum by 7 days (Lee et al., 2005). However, the last three sequential isolates (positive only for $\mathrm{C}_{4}-\mathrm{HSL}$ ) formed biofilms that consisted of irregular cell aggregates that failed to cover the entire surface (Lee et al., 2005). Consequently, the failure to develop biofilms by those three isolates may be due to the loss of either $3 \mathrm{OC}_{12}$-HSL (a non-functional las system) or twitching motility, or both. Analysis of our isolates showed that CI-2 and CI-4 are competent in their twitching motility (Schaber et al., 2004). However, neither isolate produced $\mathrm{C}_{4}$-HSL and both produced very low levels of $3 \mathrm{OC}_{12}$-HSL (Schaber et al., 2004), yet both isolates produced mature biofilms that covered the substratum more completely than PAO1 (Fig. 1, Table 1). Therefore, the development of biofilms by these isolates did not require fully functional las or $r h l \mathrm{QS}$ systems. (3) Three of the isolates described by Lee et al. (2005) showed biofilms with abnormal architecture. One isolate (65608a/1999) showed attachment and microcolony formation at day 3; however, at day 7, the biofilm developed into a structure with massive elevated perpendicular colonies (Lee et al., 2005). The architecture of CI-5 mature biofilm was somewhat similar to that produced by $65680 \mathrm{a} / 1999$ (Fig. 1). The CI-5 day 7 biofilm covered only $32.5 \%$ of the surface but contained microcolonies that reached $80 \mu \mathrm{m}$ in height (Fig. 1, Table 1). At this time, the specific factor(s) involved in the production of these abnormal biofilm architectures is not known. Neither 65680a/1999 nor CI-5 is completely defective in autoinducer production, swimming motility or twitching motility (Lee et al., 2005; Schaber et al., 2004), nor is biofilm initiation likely to be a factor in the development of this architecture. While $65680 \mathrm{a} / 1999$ showed reduced biofilm initiation, CI-5 was not significantly defective (Lee et al., 2005; Schaber et al., 2004). Furthermore, the isolation site is unlikely to be a factor: 65680a/1999 was isolated from CF sputum, whereas CI-5 was isolated from urine (Lee et al., 2005).

Our analysis suggests that the infection site may not influence biofilm formation by $P$. aeruginosa. For example, the respiratory isolate CI-2 and its sequential isolate CI-4 produced mature biofilms that covered more substratum than PAO1 (Table 1). However, another respiratory isolate, CI-3, produced biofilm that covered only $19 \%$ of the substratum (Table 1). Results reported by Lee et al. (2005) support the above possibility. Although all of the $P$. aeruginosa strains described by Lee et al. (2005) were respiratory isolates, they produced biofilms with variable structures. Whether differences in the efficiency of biofilm formation influence (directly or indirectly) the outcome of $P$. aeruginosa infection is not known. CI-2 and CI-4 were obtained from a patient on respiratory support who succumbed to $P$. aeruginosa sepsis. CI-3 on the other hand was obtained from a patient with chronic obstructive pulmonary disease who was successfully treated and discharged.

In the analysis of the mature biofilms formed by our CI, it is important to consider that while we utilized the welldifferentiated biofilm formed by PAO1 as a reference point for comparison, the CI are not isogenic mutants. Thus, the different biofilms shown in Fig. 1 and described in Table 1 may not reflect any defective phenotypes. Rather, each biofilm may represent a unique adaptation of that specific CI to the environment from which it was isolated.

\section{ACKNOWLEDGEMENTS}

The authors thank Daniel Wozniak, Wake Forest University School of Medicine, for phage F116 and the anti-PilA antibody. The authors also thank Tim Tolker-Nielsen, BioCentrum-DTU, for the pilA mutant. This study was supported by the Department of Surgery at Texas Tech University Health Sciences Center.

\section{REFERENCES}

Anderl, J. N., Zahller, J., Roe, F. \& Stewart, P. S. (2003). Role of nutrient limitation and stationary-phase existence in Klebsiella pneumoniae biofilm resistance to ampicillin and ciprofloxacin. Antimicrob Agents Chemother 47, 1251-1256.

Boles, B. R., Thoendel, M. \& Singh, P. K. (2005). Rhamnolipids mediate detachment of Pseudmonas aeruginosa from biofilms. Mol Microbiol 57, 1210-1223.

Borriello, G., Werner, E., Roe, F., Kim, A. M., Ehrlich, G. D. \& Stewart, P. S. (2004). Oxygen limitation contributes to antibiotic tolerance of Pseudomonas aeruginosa in biofilms. Antimicrob Agents Chemother 48, 2659-2664.

Carty, N. L., Rumbaugh, K. P. \& Hamood, A. N. (2003). Regulation of toxA by PtxR in Pseudomonas aeruginosa PA103. Can J Microbiol 49, 450-464.

Carty, N. L., Layland, N., Colmer-Hamood, J. A., Calfee, M. W., Pesci, E. C. \& Hamood, A. N. (2006). PtxR modulates the expression of QScontrolled virulence factors in the Pseudomonas aeruginosa strain PAO1. Mol Microbiol 61, 782-794.

Costerton, J. W., Stewart, P. S. \& Greenberg, E. P. (1999). Bacterial biofilms: a common cause of persistent infections. Science 284, 1318-1322.

Davies, D. G., Parsek, M. R., Pearson, J. P., Iglewski, B. H., Costerton, J. W. \& Greenberg, E. P. (1998). The involvement of cell-to-cell signals in the development of a bacterial biofilm. Science 280, 295-298.

De Kievit, T. R., Gillis, R. J., Marx, S., Brown, C. \& Iglewski, B. H. (2001). Quorum sensing genes in Pseudomonas aeruginosa biofilms: their role and expression patterns. Appl Environ Microbiol 67, 1865-1873.

Deziel, E., Comeau, Y. \& Villemur, R. (2001). Initiation of biofilm formation by Pseudomonas aeruginosa 57RP correlates with emergence of hyperpiliated and highly adherent phenotypic variants 
deficient in swimming, swarming, and twitching motilities. J Bacteriol 183, 1195-1204.

Donlan, R. M. \& Costerton, J. W. (2002). Biofilms: survival mechanisms of clinically relevant microorganisms. Clin Microbiol Rev 15, 167-193.

Head, N. E. \& Yu, H. (2004). Cross-sectional analysis of clinical and environmental isolates of Pseudomonas aeruginosa: biofilm formation, virulence, and genome diversity. Infect Immun 72, 133-144.

Hentzer, M., Teitzel, G. M., Balzer, G. J., Heydorn, A., Molin, S., Givskov, M. \& Parsek, M. R. (2001). Alginate overproduction affects Pseudomonas aeruginosa biofilm structure and function. J Bacteriol 183, 5395-5401.

Heydorn, A., Nielsen, A. T., Hentzer, M., Sternberg, C., Givskov, M., Ersboll, B. K. \& Molin, S. (2000). Quantification of biofilm structures by the novel computer program COMSTAT. Microbiology 146, 2395-2407.

Heydorn, A., Ersboll, B., Kato, J., Hentzer, M., Parsek, M. R., TolkerNielsen, T., Givskov, M. \& Molin, S. (2002). Statistical analysis of Pseudomonas aeruginosa biofilm development: impact of mutations in genes involved in twitching motility, cell-to-cell signaling, and stationary-phase sigma factor expression. Appl Environ Microbiol 68, 2008-2017.

Holloway, B. W., Krishnapillai, V. \& Morgan, A. F. (1979). Chromosomal genetics of Pseudomonas. Microbiol Rev 43, 73-102.

Klausen, M., Heydorn, A., Ragas, P., Lambertsen, L., AaesJorgensen, A., Molin, S. \& Tolker-Nielsen, T. (2003). Biofilm formation by Pseudomonas aeruginosa wild type, flagella and type IV pili mutants. Mol Microbiol 48, 1511-1524.

Kohler, T., Curty, L. K., Barja, F., van Delden, C. \& Pechere, J. C. (2000). Swarming of Pseudomonas aeruginosa is dependent on cell-tocell signaling and requires flagella and pili. J Bacteriol 182, 5990-5996.

Kutter, E. \& Sulakvelidze, A. (2005). Bacteriophages: Biology and Applications. Boca Raton, FL: CRC Press.

Latifi, A., Foglino, M., Tanaka, K., Williams, P. \& Lazdunski, A. (1996). A hierarchical quorum-sensing cascade in Pseudomonas aeruginosa links the transcriptional activators LasR and RhIR (VsmR) to expression of the stationary-phase sigma factor RpoS. Mol Microbiol 21, 1137-1146.

Lee, B., Haagensen, J. A., Ciofu, O., Andersen, J. B., Hoiby, N. \& Molin, S. (2005). Heterogeneity of biofilms formed by nonmucoid Pseudomonas aeruginosa isolates from patients with cystic fibrosis. $J$ Clin Microbiol 43, 5247-5255.

Martin, D. W., Holloway, B. W. \& Deretic, V. (1993). Characterization of a locus determining the mucoid status of Pseudomonas aeruginosa: $\mathrm{AlgU}$ shows sequence similarities with a Bacillus sigma factor. $J$ Bacteriol 175, 1153-1164.

Matsukawa, M. \& Greenberg, E. P. (2004). Putative exopolysaccharide synthesis genes influence Pseudomonas aeruginosa biofilm development. J Bacteriol 186, 4449-4456.

Miller, J. H. (1972). Experiments in Molecular Genetics. Cold Spring Harbor, NY: Cold Spring Harbor Laboratory.

Nicolle, L. E. (2005). Catheter-related urinary tract infection. Drugs Aging 22, 627-639.

O'Toole, G. A. \& Kolter, R. (1998a). Flagellar and twitching motility are necessary for Pseudomonas aeruginosa biofilm development. Mol Microbiol 30, 295-304.

O'Toole, G. A. \& Kolter, R. (1998b). Initiation of biofilm formation in Pseudomonas fluorescens WCS365 proceeds via multiple, convergent signalling pathways: a genetic analysis. Mol Microbiol 28, 449-461.
Pemberton, J. M. (1973). F116: a DNA bacteriophage specific for the pili of Pseudomonas aeruginosa strain PAO. Virology 55, 558-560.

Pollack, M. (2000). Pseudomonas aeruginosa. In Mandell, Douglas and Bennett's Principles and Practice of Infectious Diseases, pp. 2310-2327. Edited by G. L. Mandell, J. E. Bennett \& R. Dolin. Philadelphia, PA: Churchill Livingstone.

Prince, A. S. (2002). Biofilms, antimicrobial resistance, and airway infection. N Engl J Med 347, 1110-1111.

Roy-Burman, A., Savel, R. H., Racine, S., Swanson, B. L., Revadigar, N. S., Fujimoto, J., Sawa, T., Frank, D. W. \& Wiener-Kronish, J. P. (2001). Type III protein secretion is associated with death in lower respiratory and systemic Pseudomonas aeruginosa infections. I Infect Dis 183, 1767-1774.

Rumbaugh, K. P., Griswold, J. A. \& Hamood, A. N. (1999). Pseudomonas aeruginosa strains obtained from patients with tracheal, urinary tract and wound infection: variations in virulence factors and virulence genes. J Hosp Infect 43, 211-218.

Rumbaugh, K. P., Griswold, J. A. \& Hamood, A. N. (2000). The role of quorum sensing in the in vivo virulence of Pseudomonas aeruginosa. Microbes Infect 2, 1721-1731.

Sauer, K., Camper, A. K., Ehrlich, G. D., Costerton, J. W. \& Davies, D. G. (2002). Pseudomonas aeruginosa displays multiple phenotypes during development as a biofilm. J Bacteriol 184, 1140-1145.

Schaber, J. A., Carty, N. L., McDonald, N. A., Graham, E. D., Cheluvappa, R., Griswold, J. A. \& Hamood, A. N. (2004). Analysis of quorum sensing-deficient clinical isolates of Pseudomonas aeruginosa. J Med Microbiol 53, 841-853.

Schweizer, H. P. (1991). Escherichia-Pseudomonas shuttle vectors derived from pUC18/19. Gene 97, 109-121.

Seed, P. C., Passador, L. \& Iglewski, B. H. (1995). Activation of the Pseudomonas aeruginosa lasI gene by LasR and the Pseudomonas autoinducer PAI: an autoinduction regulatory hierarchy. J Bacteriol 177, 654-659.

Shrout, J. D., Chopp, D. L., Just, C. L., Hentzer, M., Givskov, M. \& Parsek, M. R. (2006). The impact of quorum sensing and swarming motility on Pseudomonas aeruginosa is nutritionally conditional. Mol Microbiol 62, 1264-1277.

Smith, A. W. \& Iglewski, B. H. (1989). Transformation of Pseudomonas aeruginosa by electroporation. Nucleic Acids Res 17, 10509.

Stewart, P. S. \& Costerton, J. W. (2001). Antibiotic resistance of bacteria in biofilms. Lancet 358, 135-138.

Stewart, P. S., Peyton, B. M., Drury, W. J. \& Murga, R. (1993). Quantitative observations of heterogeneities in Pseudomonas aeruginosa biofilms. Appl Environ Microbiol 59, 327-329.

Stickler, D. J. (2002). Susceptibility of antibiotic-resistant Gramnegative bacteria to biocides: a perspective from the study of catheter biofilms. J Appl Microbiol 92 (Suppl), 163S-170S.

van Delden, C. \& Iglewski, B. H. (1998). Cell-to-cell signaling and Pseudomonas aeruginosa infections. Emerg Infect Dis 4, 551-560.

Venturi, V. (2006). Regulation of quorum sensing in Pseudomonas. FEMS Microbiol Rev 30, 274-291.

Walters, M. C., III, Roe, F., Bugnicourt, A., Franklin, M. J. \& Stewart, P. S. (2003). Contributions of antibiotic penetration, oxygen limitation, and low metabolic activity to tolerance of Pseudomonas aeruginosa biofilms to ciprofloxacin and tobramycin. Antimicrob Agents Chemother 47, 317-323. 